\title{
THE H-PRINCIPLE AND PSEUDOCONCAVE CR MANIFOLDS
}

\author{
C. DENSON HILL AND EGMONT PORTEN
}

\begin{abstract}
The H-principle, which is the analogue, for CR manifolds, of the classical Hartogs principle in several complex variables, is known to be valid in the small on a pseudoconcave CR manifold of any codimension. However it fails in the large, as has been shown by the counterexample found in [HN1]. Hence there is an underlying obstruction to the global $\mathrm{H}$-principle on a pseudoconcave $\mathrm{CR}$ manifold. The purpose of this note is to take the first steps toward a deeper understanding of this obstruction.
\end{abstract}

\section{INTRODUCTION}

Consider a smooth CR manifold $M$ of type $(n, k)$ which is a generic CR submanifold of a complex manifold $X$. For a detailed discussion of all basic concepts concerning CR manifolds, see any one of [HN1, [HN2, [HN3], HN4]. Here $n$ is the CR dimension, $k$ is the CR codimension, so $\operatorname{dim}_{\mathbb{R}} M=2 n+k$, and $M$ being generic in $X$ implies that $\operatorname{dim}_{\mathbb{C}} X=n+k$. When $k=0, M$ is just an $n$-dimensional complex manifold, and we take $X=M$. In this note we shall always be considering the following situation: We assume that $\Omega$ is a connected open set in $M, K$ is a compact set in $\Omega$, and $\Omega \backslash K$ is connected and nonvoid. $C R(D)$ denotes the algebra of continuous $\mathrm{CR}$ functions in the open set $D \subset M$, i.e., functions which satisfy the tangential Cauchy-Riemann equations on $M$ in the sense of distributions. When $k=0$ the CR functions are simply the holomorphic functions, and we write $\mathcal{O}(D)$ instead of $C R(D)$.

In this situation we say that the H-principle holds for the pair $(\Omega, K)$ iff the restriction map

$$
C R(\Omega) \longrightarrow C R(\Omega \backslash K)
$$

is surjective. Here $K$ should be thought of as a "hole" which can always be "filled" by $\mathrm{CR}$ functions. If the $\mathrm{CR}$ functions on $M$ enjoy the weak unique continuation property (i.e., vanishing in an open set implies vanishing everywhere), then the restriction map (1.1) is injective, so in that case the validity of the H-principle actually means that there is an isomorphism $C R(\Omega) \cong C R(\Omega \backslash K)$ of algebras.

When $k=0$, (1.1) becomes $\mathcal{O}(\Omega) \longrightarrow \mathcal{O}(\Omega \backslash K)$. If this map is surjective, then it is an isomorphism, and the hole $K$ in $\Omega$ can always be filled by holomorphic extension; this is the classical Hartogs-principle (H-principle) in several complex variables. The classical Hartogs-principle is valid for any pair $(\Omega, K)$, provided the $n$-dimensional complex manifold $M$ is $(n-2)$-complete (in particular when $n>1$, and $M$ is $\mathbb{C}^{n}$, or $M$ is Stein), for example see $[\mathrm{AH}$.

Date: August 25, 2021 
When $k \geq 1$ the H-principle clearly fails, as is seen by easy counterexamples, unless $M$ has some amount of pseudoconcavity (perhaps in a weak sense). A CR manifold $M$ is said to be $q$-pseudoconcave provided that the Levi form $\mathcal{L}_{x_{0}}(\xi, \cdot)$ of $M$ at $x_{0}$ has at least $q$ negative eigenvalues in each nonzero characteristic conormal direction $\xi$ at $x_{0}$, for every $x_{0} \in M$.

A 1-pseudoconcave CR manifold is simply called pseudoconcave. A pseudoconcave CR manifold $M$ enjoys the property that to any connected open set $D \subset M$, one can associate a connected open set $\tilde{D} \subset X$, with $D=\tilde{D} \cap M$, such that the restriction map $\mathcal{O}(\tilde{D}) \rightarrow C R(D)$ is an isomorphism of algebras (see [BP], [NV], HN5]); so in particular, the CR functions on $M$ have the strong unique continuation property (i.e., all derivatives vanishing at a point implies vanishing everywhere), and are as smooth as $M$ is. So in this situation the CR functions behave very much like holomorphic functions. Thus on a pseudoconcave CR manifold $M$, it seems to be a natural question to ask: When does the H-principle hold for certain pairs $(\Omega, K)$ ?

Indeed it was shown in $\mathrm{He}$ that the $\mathrm{H}$-principle is valid on a smooth pseudoconcave CR manifold $M$ for any pair $(\Omega, K)$, provided that $\Omega$ is sufficiently small. For real analytic $M$ and real analytic CR functions, this result has been extended $[\mathrm{HeM}$ to the weakly 1-pseudoconcave case. See also $\mathrm{Na}$. for an earlier result, in a special case.

On the other hand a very simple counterexample was found in HN1 for the situation where $K$ becomes too big: Take $M$ to be the pseudoconcave CR hypersurface of type $(2,1)$ in $\mathbb{C}^{3}$ given by

$$
M:\left|z_{1}\right|^{2}+\left|z_{2}\right|^{2}=1+\left|z_{3}\right|^{2},
$$

and $K=S^{3}$ to be $M \cap\left\{z_{3}=0\right\}$. By considering the CR function $1 / z_{3}$ on $M \backslash S^{3}$, one sees that the H-principle cannot hold for any pair $\left(\Omega, S^{3}\right)$. Thus there is an underlying obstruction to the global H-principle for pseudoconcave $M$. The present note is intended as a first step toward a deeper understanding of this obstruction.

We shall show below that example (1.2) has some interesting features:

(A): If $S^{3}$ is replaced by $K_{\omega}=S^{3} \backslash \omega$, where $\omega$ is an arbitrarily small nonempty open set on $S^{3}$, then the H-principle holds for any pair $\left(\Omega, K_{\omega}\right)$.

(B): If $S^{3}$ is replaced by $K=\tilde{S}^{3}$, where $\tilde{S}^{3} \subset M$ is a small, but randomly chosen smooth perturbation of $S^{3}$, then the H-principle holds for any pair $\left(\Omega, \tilde{S}^{3}\right)$.

We also obtain that both $C R\left(M \backslash K_{\omega}\right)$ and $C R\left(M \backslash \tilde{S}^{3}\right)$ are isomorphic to $\mathcal{O}\left(\mathbb{C}^{3}\right)$, the space of entire functions on $\mathbb{C}^{3}$.

Note that in both (A) and (B) above, the hole which is filled by the Hprinciple is "thin", in the sense that both $K_{\omega}$ and $\tilde{S}^{3}$ have codimension 2 in $M$. One can ask about trying to fill "thick" holes, by which we mean that $K$ should be a compact set having non-empty interior. In this respect (A) and (B) behave quite differently. 
(C): Given a pair $\left(\Omega, K_{\omega}\right)$, there exists an $\epsilon=\epsilon\left(\Omega, K_{\omega}\right)>0$ such that the H-principle holds for any $\left(\Omega, K_{\omega}^{\epsilon}\right)$, where $K_{\omega}^{\epsilon}$ denotes the closed $\epsilon$ neighborhood of $K_{\omega}$ in $M$.

(D): But if $\tilde{S}^{3}$ in (B) is replaced by any slender compact neighborhood $\tilde{K}$ of $\tilde{S}^{3}$ in $M$ which contains the original $S^{3}$, then the H-principle cannot hold for any pair $(\Omega, \tilde{K})$, as is obvious because of the original example.

We should also draw the readers attention to the interesting papers [L, [LL1, [LL2, B], in which global results are obtained under additional assumptions, mainly on the degree of pseudoconcavity.

The first author would like to acknowledge the support of the Mathematics Institute of Humboldt University in Berlin, and in particular the kind hospitality of Professor Jürgen Leiterer.

\section{Elementary use of analytic discs.}

In order to motivate the general results in section 3 , we first discuss points (A) and (C) for example (1.2).

We consider parameters $(r, s)$ with $0 \leq r<\infty$ and $s \in S^{3}$, where $S^{3}$ denotes the unit sphere in $\mathbb{C}^{2}$ with coordinates $\left(z_{1}, z_{2}\right)$. Let $\Delta_{r, s}$ denote the subset of $\mathbb{C}^{3}$ defined by

$$
\Delta_{r, s}=\left\{\left(\left[1+r^{2}\right]^{1 / 2} s, z_{3}\right)|| z_{3} \mid<r\right\},
$$

and $\partial \Delta_{r, s}=\bar{\Delta}_{r, s} \backslash \Delta_{r, s}$ denote its boundary. Note that the $\Delta_{r, s}$ give a foliation of the exterior $\left\{\left|z_{1}\right|^{2}+\left|z_{2}\right|^{2}>1+\left|z_{3}\right|^{2}\right\}$ of our $M$ given in (1.2), the boundaries $\partial \Delta_{r, s}$ lie on $M$, and shrink to the points $(s, 0)$ when $r=0$.

In order to verify statement (A) we choose an open set $V$ on $S^{3}$ such that $K_{\omega} \subset V \subset \subset S^{3} \cap \Omega$, and $r_{2}>0$ sufficiently small so that

$$
\bigcup_{0<r<r_{2}, s \in V} \partial \Delta_{r, s} \subset \Omega
$$

Fix $(\hat{r}, \hat{s}) \in\left(0, r_{2}\right) \times V$ and choose in $V$ a continuous path $\gamma(t), 0 \leq t \leq 1$, connecting $\hat{s}$ with some $\tilde{s} \in V \backslash K_{\omega}$. Then for $0 \leq t \leq 1$ the boundaries $\partial \Delta_{\hat{r}, \gamma(t)} \subset \Omega \backslash K_{\omega}$. But by shrinking $\hat{r}$ to zero, the boundary $\partial \Delta_{\hat{r}, s}$ can be contracted to the point $\tilde{s} \in \Omega \backslash K_{\omega}$. As was mentioned in the introduction, the CR functions on $\Omega \backslash K_{\omega}$ have holomorphic extensions to an ambient neighborhood of $\Omega \backslash K_{\omega}$ in $\mathbb{C}^{3}$. By the Kontinuitätssatz we therefore obtain holomorphic extension to

$$
\bigcup_{0<r<r_{2}, s \in V} \Delta_{r, s} \subset \mathbb{C}^{3},
$$

which is an open set attached to an open neighborhood of $K_{\omega}$ in $M$, from the exterior. By the pseudoconcavity of $M$ we obtain holomorphic extension across $K_{\omega}$, proving $(\mathrm{A})$.

In order to prove $(\mathrm{C})$ for the pair $\left(\Omega, K_{\omega}^{\epsilon}\right)$ it suffices to modify the above argument as follows: With $V$ and $r_{2}$ chosen exactly as above, we first remark that the same proof works if $K_{\omega}$ is replaced by $K_{\omega, \delta}$, where $K_{\omega, \delta}$ is the closed $\delta$-neighborhood of $K_{\omega}$ in $S^{3}$, and $\delta>0$ is sufficiently small. In fact the envelope of 
holomorphy of the set (2.3) still contains $K_{\omega, \delta}$. Next we observe that if $r_{1}>0$ is chosen sufficiently small, then the envelope of holomorphy of

$$
\bigcup_{r_{1}<r<r_{2}, s \in V} \partial \Delta_{r, s} \subset \Omega,
$$

contains a neighborhood $W$ of $K_{\omega, \delta}$ in $M$. Hence it suffices to choose $\epsilon>0$ so small that every function in $\mathcal{O}\left(\Omega \backslash K_{\omega}^{\epsilon}\right)$ extends holomorphically to the set (2.4) and that $K_{\omega}^{\epsilon} \subset W$. This completes the proof of point (C).

A direct proof of (B) would be considerably more involved. However we obtain it as a special case of Theorem 3.1 in the next section.

\section{REMOVING THIN SINGUlaRities}

In this section we return to the general situation of a CR manifold $M$ of type $(n, k)$, as in the beginning of the Introduction, and we study the H-principle for thin holes $K$.

Consider a point $x_{0} \in M$ and a local system $z=\left(z_{1}, z_{2}, \ldots, z_{n+k}\right)$ of holomorphic coordinates for $X$, centered at $x_{0}$. Then $M$ is locally defined by

$$
M=\left\{\rho_{1}(z)=0, \rho_{2}(z)=0, \ldots, \rho_{k}(z)=0\right\}
$$

where the $\rho_{i}$ are smooth real valued functions, defined in a neighborhood of $x_{0}$. The fact that $M$ is generic in $X$ means that $\bar{\partial} \rho_{1}, \bar{\partial} \rho_{2}, \ldots, \bar{\partial} \rho_{k}$ are linearly independent at $x_{0}$. The holomorphic tangent space to $M$ at $x_{0}$ is defined by

$$
H_{x_{0}} M=\left\{w \in \mathbb{C}^{n+k} \mid \sum_{j=1}^{n+k} \frac{\partial \rho_{i}\left(x_{0}\right)}{\partial z_{j}} w_{j}=0 ; i=1,2, \ldots, k\right\} .
$$

The Levi form $\mathcal{L}_{x_{0}}(\xi, \cdot)$ of $M$ at $x_{0}$, in the characteristic covector direction $\xi=$ $\left(\xi_{1}, \xi_{2}, \ldots, \xi_{k}\right)$, can be written as

$$
\sum_{i=1}^{k} \sum_{j, l=1}^{n+k} \xi_{i} \frac{\partial^{2} \rho_{i}\left(x_{0}\right)}{\partial z_{j} \partial \bar{z}_{l}} w_{j} \bar{w}_{l} .
$$

It is a hermitian form on $H_{x_{0}} M \cong \mathbb{C}^{n}$, and it is useful to write $T_{x_{0}} M \cong \mathbb{C}^{n} \times \mathbb{R}^{k}$.

Next let $N$ be a smooth closed submanifold of $M$ having real codimension 2 in $M$. Then $N$ is locally defined by

$$
N=\left\{\rho_{1}(z)=0, \ldots, \rho_{k}(z)=0, \rho_{k+1}(z)=0, \rho_{k+2}(z)=0\right\}
$$

in a neighborhood of $x_{0} \in N$, with $d \rho_{1}, \ldots, d \rho_{k}, d \rho_{k+1}, d \rho_{k+2}$ linearly independent at $x_{0}$. Since we are not assuming that $N$ is a CR manifold, the

$$
\operatorname{span}\left\{\bar{\partial} \rho_{1}, \ldots, \bar{\partial} \rho_{k}, \bar{\partial} \rho_{k+1}, \bar{\partial} \rho_{k+2}\right\}
$$

at $x_{0}$ may have dimension $k+2, k+1$ or $k$. Thus the 3 possibilities are $T_{x_{0}} N \cong$ $\mathbb{C}^{n-2} \times \mathbb{R}^{k+2}, T_{x_{0}} N \cong \mathbb{C}^{n-1} \times \mathbb{R}^{k}$ or $T_{x_{0}} N \cong \mathbb{C}^{n} \times \mathbb{R}^{k-2}$. In the case where $T_{x_{0}} N \cong \mathbb{C}^{n-2} \times \mathbb{R}^{k+2}, N$ is said to be generic at $x_{0}$.

In the theorem below we consider a smooth CR manifold $M$ of type $(n, k)$, as in the Introduction, and a smooth closed connected submanifold $N$ of $M$. 
Theorem 3.1. Assume $M$ is pseudoconcave, $N$ has real codimension 2 in $M$, and let $K$ be a compact subset of $N$. Then the H-principle is valid for any pair $(\Omega, K)$ provided one of the following conditions holds:

(a) $K$ is a proper subset of $N$, or

(b) $K=N$ and there is a point $x_{0} \in N$ at which $N$ is generic.

Let us interpret Theorem 3.1 in terms of the pseudoconcave $M$ of type $(2,1)$ in example (1.2): If we choose $N=S^{3}$, then from (a) we recover our statement (A). However (a) gives much more; namely it yields an analogous result for any smooth 3-dimensional submanifold $N$ of $M$.

If we choose $N=\tilde{S}^{3}$, then from (b) we obtain our remaining statement (B). This is because a randomly chosen deformation of $S^{3}$ will contain many generic points. However (b) gives much more; namely any codimension 2 counterexample $K=N$ has to be a CR manifold of type $(1,1)$.

Proof of Theorem 3.1. Because of the uniqueness of CR extension on $M$, it will suffice to show that we have CR extension across any given point $x_{0} \in K$. As was mentioned in the Introduction, any given $f \in C R(\Omega \backslash K)$ has a holomorphic extension, again denoted by $f$, to an open set $\tilde{D}$ in $X$ with $\Omega \backslash K=\tilde{D} \cap M$. By [MP1, Theorem 4], see also [CS, [J], and [M], there is an open truncated wedge $W \subset X$ attached to an open neighborhood $U$ of $x_{0}$ in $M$, an open set $\hat{D}$ of $X$ with $\hat{D} \subset \tilde{D}$ and with $\Omega \backslash K=\hat{D} \cap M$, and an $f_{w} \in \mathcal{O}(W)$ such that $\left.f_{w}\right|_{W \cap \hat{D}}=\left.f\right|_{W \cap \hat{D}}$. Here $W$ and $\hat{D}$ do not depend on $f$.

We have to justify that the theorem being used here applies under either hypothesis (a) or (b). First we observe that in both cases $M$ and $M \backslash K$ are globally minimal. Indeed, being pseudoconcave, $M$ is even minimal in each of its points. This can be seen as follows: In each $p \in M$, the brackets of the vectorfields tangent to $H$ span the whole tangent space $T_{p} M$. Since these brackets are tangent to the local CR orbit of $M$ in $p$, the local CR orbit has to be open (see [S], J], MP1 for detailed information on CR orbits). In case (b) we also need $n \geq 2$, which is an obvious consequence of pseudoconcavity.

In terms of the local holomorphic coordinates $z$ in $\mathbb{C}^{n+k}$, centered at $x_{0}, W$ can be chosen as follows: For some open truncated cone $C \subset \mathbb{C}^{n+k}$, with vertex at the origin, $W=U+C=\{z+c \mid z \in U, c \in C\}$. However associated to the open set $U$ in $M$ there is an open set $\tilde{U}$ in $\mathbb{C}^{n+k}$, with $U=\tilde{U} \cap M$, such that $\mathcal{O}(\tilde{U}) \cong C R(U)$. For any vector $c \in C$ we define the rigid motion translates of $U$ by $U_{c}=U+c$, and note that $U_{c} \subset W$. Note that $\mathcal{O}\left(\tilde{U}_{c}\right) \cong C R\left(U_{c}\right)$ where $\tilde{U}_{c}=\tilde{U}+c$. By choosing $|c|$ sufficiently small, we have $x_{0} \in \tilde{U}_{c}$. This gives the desired extension of our original $f$ to a neighborhood of $x_{0}$, and completes the proof of the theorem.

We end this section with a second theorem which shows that for pseudoconcave CR manifolds $M$ the $\mathrm{H}$-principle can only fail for holes $K$ having codimension at most 2. Let $\mathcal{H}^{s}(K)$ denote s-dimensional Hausdorff measure of $K$.

Theorem 3.2. Assume $M$ as in Theorem 3.1, and let $K \subset M$ be any compact subset with $\mathcal{H}^{2 n+k-2}(K)=0$. Then the H-principle is valid for any pair $(\Omega, K)$. 
Proof. The proof is the same as that of Theorem 3.1 except that we use [MP2, Theorem 1.1], see also [LS], [CS], to obtain holomorphic extension into a wedge $W$ attached to a neighborhood of $x_{0} \in K$.

\section{Revisiting the example}

We now return to the discussion of the specific $M$ given by (1.2). We know from (B) that for a pair $\left(M, \tilde{S}^{3}\right)$ the H-principle is valid for most small deformations $\tilde{S}^{3}$ of $S^{3}$. In the next theorem we characterize precisely those small deformations for which the H-principle for $\left(M, \tilde{S}^{3}\right)$ fails.

Theorem 4.1. Let $\tilde{S}^{3}$ be a sufficiently small $\mathcal{C}^{2}$-deformation of $S^{3}$. Then the $H$ principle for $\left(M, \tilde{S}^{3}\right)$ fails if and only if $\tilde{S}^{3}$ is the intersection of $M$ with a complex hypersurface $Y$ in $\mathbb{C}^{3}$.

Proof. If there is such a $Y$, then there is an entire function $g$ on $\mathbb{C}^{3}$ such that $Y=\left\{z \in \mathbb{C}^{3} \mid g(z)=0\right\}$. Then $1 / g$ is a CR function on $M \backslash \tilde{S}^{3}$ which has no CR extension across $\tilde{S}^{3}$, so the H-principle fails.

On the other hand, if the H-principle fails, then we know that $\tilde{S}^{3}$ must be a CR manifold of type $(1,1)$. Let $\pi: \mathbb{C}^{3} \rightarrow \mathbb{C}^{2}$ and $\pi_{3}: \mathbb{C}^{3} \rightarrow \mathbb{C}$ denote the holomorphic projections onto the $\left(z_{1}, z_{2}\right)$-plane and the $z_{3}$-axis, respectively. Since the deformation is $\mathcal{C}^{2}$-small, we obtain $\tilde{S}^{3}$ as a graph of the CR function $\phi=\pi_{3}$ 。 $\left(\left.\pi\right|_{\tilde{S}^{3}}\right)^{-1}$ over the stricly convex 3 -dimensional hypersurface $\pi\left(\tilde{S}^{3}\right)$, which bounds a domain $G$ in $\mathbb{C}^{2}$. It is well known that $\phi$ has an extension $\tilde{\phi} \in \mathcal{O}(G) \cap \mathcal{C}^{2}(\bar{G})$. The graph of $\tilde{\phi}$ over $G$ defines a smooth complex hypersurface $\tilde{Y}$ bounded by $\tilde{S}^{3}$. Note that $\tilde{Y}$ is contained in the interior of the domain $\mathcal{D}=\left\{\left|z_{1}\right|^{2}+\left|z_{2}\right|^{2}<1+\left|z_{3}\right|^{2}\right\}$ and is transversal to its boundary $M$.

Next we show that any $f \in C R\left(M \backslash \tilde{S}^{3}\right)$ has a holomorphic extension $\tilde{f}$ to $\mathcal{D} \backslash \tilde{Y}$. Let $\epsilon>0$ be given. By Runge approximation there is a polynomial $P_{\epsilon} \in$ $\mathbb{C}\left[z_{1}, z_{2}\right]$ such that $\max _{\bar{G}}|P-\tilde{\phi}|<\epsilon$. Then the complex hypersurface

$$
Y_{\epsilon, c}=\left\{z_{3}=P_{\epsilon}\left(z_{1}, z_{2}\right)+c\right\}
$$

does not intersect $\tilde{Y}$ provided $|c|>\epsilon$. Since each $Y_{\epsilon, c}$ is Stein, the H-principle implies that $f$ has a holomorphic extension to $\overline{\mathcal{D}} \cap Y_{\epsilon, c}$ for $|c|>\epsilon$, and we obtain the holomorphic extension of $f$ to

$$
\mathcal{D}_{\epsilon}=\mathcal{D} \cap\left\{\left|z_{3}-P_{\epsilon}\left(z_{1}, z_{2}\right)\right|>\epsilon\right\} .
$$

Taking $\epsilon \searrow 0$ we see that $f$ has a holomorphic extension to $\mathcal{D} \backslash \tilde{Y}$.

By $[\mathrm{D}$, see also $[\mathrm{P}$, there are only two possibilities for the envelope of holomorphy $\Sigma$ of $\mathcal{D} \backslash \tilde{Y}$ : Either (1) $\Sigma=\mathbb{C}^{3}$ or else (2) there is a complex hypersurface $Y$ of $\mathbb{C}^{3}$ satisfying $\tilde{Y}=Y \cap \mathcal{D}$ such that $\Sigma=\mathbb{C}^{3} \backslash Y$. Here we have used that the envelope of holomorphy of $\mathcal{D}$ is all of $\mathbb{C}^{3}$. Possibility (1) is ruled out by our hypothesis. Thus it remains only to verify that $Y \cap M=\tilde{S}^{3}$. Obviously $\tilde{S}^{3} \subset Y \cap M$. But there are no points in $\left(M \backslash \tilde{S}^{3}\right) \cap Y$ because $M$ is pseudoconcave. This completes the proof of the theorem. 
Remarks. It follows from the argument above, that if the H-principle fails for $\left(M, \tilde{S}^{3}\right)$, where $\tilde{S}^{3} \subset M$ is a small $\mathcal{C}^{2}$-deformation of $S^{3}$, then $\tilde{S}^{3}$ must be a real analytic CR manifold of type $(1,1)$.

Let $\Omega$ be a given open set on $M$ containing $S^{3}$. Again consider $\tilde{S}^{3} \subset \Omega$ to be a sufficiently small $\mathcal{C}^{2}$-deformation of $S^{3}$. If the H-principle fails for $\left(\Omega, \tilde{S}^{3}\right)$ then, once again $\tilde{S}^{3}$ must be a real analytic $\mathrm{CR}$ manifold of type $(1,1)$. This follows by a straightforward modification of the proof of Theorem 4.1, in which the role of $\mathbb{C}^{3}$ is replaced by the envelope of holomorphy of an appropriate domain, analogous to $D \backslash \tilde{Y}$.

\section{REFERENCES}

[AH] A. Andreotti, C. D. Hill: E. E. Levi convexity and the Hans Lewy problem I: Reduction to vanishing theorems. Annali Scuola Norm. Sup. Pisa 26 (1972), 747-806.

[BP] A. Boggess, J. Polking: Holomorphic extension of CR functions. Duke Math. J. 49 (1982), 757-784.

[B] J. Brinkschulte: The Hartogs phenomenon in hypersurfaces with constant signature. Prépublication de l'Institut Fourier 564 (2002).

[CS] E. M. Chirka, E.-L. Stout: Removable singularities in the boundary. Contributions to Complex Analysis and Analytic Geometry, Aspects of Mathematics E 26, Vieweg 1994, 43-104.

[D] G. Dloussky: Enveloppes d'holomorphie et prolongement d'hypersurfaces. Lecture Notes in Math. 578, Springer-Verlag 1977, 217-235.

[He] G. M. Henkin: The Hartogs-Bochner effect on CR-manifolds. Soviet Math. Dokl. 29 (1984), 78-82.

[HeM] G. M. Henkin, V. Michel: Principe de Hartogs dans les variétés CR. Preprint 2002.

[HN1] C. D. Hill, M. Nacinovich: Pseudoconcave CR manifolds. V. Ancona, E. Ballico, and A. Silva, Eds., Marcel Dekker, 1996, 275-298.

[HN2] C. D. Hill, M. Nacinovich: Aneurysms of pseudoconcave CR manifolds. Math. Z. 220 (1995), 347-367.

[HN3] C. D. Hill, M. Nacinovich: Duality and distribution cohomology for CR manifolds. Annali Scuola Norm. Sup. Pisa XXII (1995), 315-339.

[HN4] C. D. Hill, M. Nacinovich: On the Cauchy problem in complex analysis. Ann. Mat. Pura Appl. CLXXI (IV) (1996), 159-179.

[HN5] C. D. Hill, M. Nacinovich: Conormal suspensions of differential complexes. J. Geom. Anal. 10 (2000), 481-523.

[J] B. Jöricke: Boundaries of singularity sets, removable singularities, and CR-invariant subsets of CR-manifolds, J. Geom. Anal. 6 (1996), 555-611.

[L] C. Laurent-Thiébaut: Résolution $d u \bar{\partial}$ à support compact et phénomène de HartogsBochner dans les variétés CR. Proc. Symp. Pure. Math. 52 (1991), 239-249.

[LL1] C. Laurent-Thiébaut, J. Leiterer: On the Hartogs-Bochner extension phenomenon for differential forms. Math. Ann. 284 (1989), 103-119.

[LL2] C. Laurent-Thiébaut, J. Leiterer: Dolbeault isomorphism for CR manifolds. Math. Ann. 325 (2003), 165-185.

[LS] G. Lupacciolu, E.-L. Stout: Removable singularities for $\bar{\partial}_{b}$. Proceedings of the Mittag Leffler Special Year in Complex Analysis, Princeton University Press, Princeton, NJ, 1993.

[M] J. Merker: On removable singularities for CR functions in higher codimension. Int. Math. Res. Not. 1 (1997), 21-56.

[MP1] J. Merker and E. Porten: On removable singularities for integrable CR-functions. Indiana U. Math. J. 48 (1999), 805-856.

[MP2] J. Merker and E. Porten: On wedge extendability of CR-meromorphic functions. Math. Z. 241 (2002), 485-512.

[NV] M. Nacinovich, G. Valli: Tangential Cauchy-Riemann complexes on distributions. Ann. Mat. Pura Appl. 146 (1987), 123-160. 
[Na] I. Naruki: Localization principle for differential complexes and its applications. Publ. Res. Inst. Math. Sc. Kyoto Univ. 8 (1972), 43-110.

[P] E. Porten: On the Hartogs-phenomenon and extension of analytic hypersurfaces in nonseparated Riemann domains. Complex Variables 47 (2002), 325-332.

[S] H. J. Sussmann: Orbits of families of vector fields and integrability of distributions.. Trans. Amer. Math. Soc. 180 (1973), 171-188.

C. Denson Hill

Department of Mathematics

Stony Brook University

Stony Brook, N.Y. 11794, USA

dhill@math.sunysb.edu

Egmont Porten

Mathematisches Institut

Humboldt-Universität zu Berlin

Rudower Chaussee 25

12489 Berlin, Germany

egmont@mathematik.hu-berlin.de 